\title{
Distillation of Bose-Einstein condensates in a double-well potential
}

\author{
Y. Shin, M. Saba, A. Schirotzek, T. A. Pasquini, A. E. Leanhardt, D. E. Pritchard, and W. Ketterle* \\ Department of Physics, MIT-Harvard Center for Ultracold Atoms, and Research Laboratory of Electronics, \\ Massachusetts Institute of Technology, Cambridge, Massachusetts, 02139
}

(Dated: November 9, 2018)

\begin{abstract}
Bose-Einstein condensates of sodium atoms, prepared in an optical dipole trap, were distilled into a second empty dipole trap adjacent to the first one. The distillation was driven by thermal atoms spilling over the potential barrier separating the two wells and then forming a new condensate. This process serves as a model system for metastability in condensates, provides a test for quantum kinetic theories of condensate formation, and also represents a novel technique for creating or replenishing condensates in new locations.

PACS numbers: 03.75.-b, 03.75.Lm, 64.60.My
\end{abstract}

The characteristic feature of Bose-Einstein condensation is the accumulation of a macroscopic number of particles in the lowest quantum state. Condensate fragmentation, the macroscopic occupation of two or more quantum states, is usually prevented by interactions [1], but may happen in spinor condensates 2, 3]. However, multiple condensates may exist in metastable situations. Let's assume that an equilibrium condensate has formed in one quantum state, but now we modify the system allowing for one even lower state. How does the original condensate realize that it is in the wrong state and eventually migrate to the true ground state of the system? What determines the time scale for this equilibration process? This is the situation which we experimentally explore in this paper using a double-well potential.

The process we study is relevant for at least four different questions. (1) The description of the formation of the condensate is a current theoretical frontier and requires finite-temperature quantum kinetic theories. There are still discrepancies between theoretical predictions and experimental results [4, 5]. Our double-well system has the advantage of being an almost closed system (little evaporation) with well defined initial conditions and widely adjustable time scales (through the height of the barrier). (2) Spinor condensates show rich ground states and collective excitations due to the multi-component order parameter 2]. Several groups have observed longlived metastable configurations $[\underline{6}, \mathbf{7},[\underline{8},[9]$ and speculated about transport of atoms from one domain to another via the thermal cloud [6, 8]. The double-well potential allows us to characterize such distillation processes in their simplest realization. (3) The incoherent transport observed here in a double well-potential imposes stringent limitations on future experiments aiming at the observation of coherent transport in Josephson junctions 10, 11, 12. (4) Our observation of condensate growth in one potential well due to the addition of thermal atoms realizes the key ideas of proposals on how to achieve a continuous atom laser [13] which is different from the experiment where condensates were replenished with transported condensates [14].

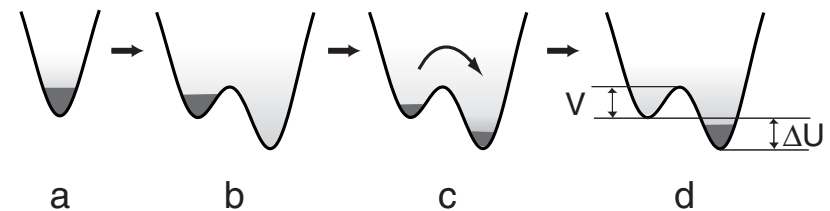

FIG. 1: Scheme for distillation of condensates in a double-well potential. (a) Condensates are loaded into the left well. (b) A new ground state is created by linearly ramping the trap depth of the right well from zero to the final value. (c) Atoms transfer into the right well via high-energy thermal atoms, and a new condensate starts to form in the right well. (d) The whole system has equilibrated. $V$ denotes the height of the potential barrier between the two wells, which is measured with respect to the bottom of the left well, and $\Delta U$ the trap depth difference between the two wells.

The scheme of the experiment is shown in Fig 1 BoseEinstein condensates in an optical dipole trap were prepared in a metastable state by creating a second trap horizontally adjacent to the first. Since the probability of quantum tunneling through the barrier was extremely small [15], the coupling between the two wells occurred only by the incoherent transfer of high-energy thermal atoms over the potential barrier between the two wells. The second trap was filled first by thermal atoms, which then formed a new condensate. By monitoring the time evolution of the double-well system we characterized how differences in chemical potential and the height of the barrier determined the dynamics.

Bose-Einstein condensates containing over $10^{7}{ }^{23} \mathrm{Na}$ atoms were created in the $\left|F=1, m_{F}=-1\right\rangle$ state in a magnetic trap, captured in the focus of a $1064 \mathrm{~nm}$ optical tweezers laser beam, and transferred into an separate "science" chamber as described in Ref. 16]. In the science chamber the condensate was transferred from the optical tweezers into another optical trap formed by a counter-propagating, orthogonally-polarized $1064 \mathrm{~nm}$ laser beam. As in Ref. [17], the double-well potential was created by passing a collimated laser beam through an acousto-optic modulator (AOM) that was driven by 
two radio frequency (rf) signals. The separation between the potential wells, $d$, was proportional to the frequency difference, and the individual trap depth was tailored by controlling the rf power at the two frequencies. Typical parameters were an $1 / e^{2}$ radius of each focused beam of $11.3 \mu \mathrm{m}$, a single-well potential depth of $U=k_{B} \times 2.4 \mu \mathrm{K}$, where $k_{B}$ is the Boltzmann constant, and a radial (axial) trap frequency, $f_{r}=830 \mathrm{~Hz}$ $\left(f_{z}=12.4 \mathrm{~Hz}\right)$. As shown in Fig 1] condensates were initially loaded into the left well with depth $U_{L}$ while the trap depth of the right well, $U_{R}$, was maintained at zero. After holding the condensates for $2 \mathrm{~s}$ to damp excitations which might have been caused by the loading process, the temperature was $T_{i}=(180 \pm 90) \mathrm{nK}$, the number of condensed atoms $N_{i}=(1.1 \pm 0.1) \times 10^{6}$ with a peak mean field energy of $\tilde{\mu}_{0} \approx k_{B} \times 300 \mathrm{nK}$, and the lifetime $\tau=(12.1 \pm 1.5) \mathrm{s}$.

The potential was transformed into a double-well potential by linearly ramping the right well potential from zero to the final value of $U_{R}$ over $500 \mathrm{~ms}$ while keeping $U_{L}$ constant. This time scale was chosen to be much longer than the radial trap period of $\sim 1 \mathrm{~ms}$ to avoid excitations. The resulting double-well potential is characterized by the trap depth difference between the two wells, $\Delta U=U_{R}-U_{L}$, and the height of the potential barrier between the two wells, $V$, which is measured with respect to the bottom of the left well, i.e. the well initially full of atoms. The barrier height was set higher than the peak atomic mean field energy of condensates so that condensed atoms remained confined to the left well during the transformation.

The thermal relaxation process was observed by taking absorption images of clouds confined in the double-well potential for various hold times after turning on the right well. In order to fully resolve the clouds in the two wells, their distance was increased to $d=31.2 \mu \mathrm{m}$ just before taking absorption images. We assume that this did not change either the number of atoms in each well or the axial density distributions, since this additional separation was done in $10 \mathrm{~ms}$, which is much shorter than the axial trap period of $\sim 100 \mathrm{~ms}$, and the height of the potential barrier exponentially increases when the two wells move apart.

Fig. 2 shows the dynamical evolution for a situation where the right well was much deeper than the left well. In that case, condensates that initially existed only in the left well were almost completely distilled within $3 \mathrm{~s}$ to form condensates of comparable size in the right well.

The time evolution of the double-well system was characterized by monitoring the number of condensed atoms and the temperature of clouds in each well. These numbers were obtained by fitting radially-integrated onedimensional atomic density cross sections to a bimodal distribution. The assumption of local equilibrium in each well is justified by a short collision time $\tau_{\text {col }} \approx 1 \mathrm{~ms}$. For the condensate, we used a Thomas-Fermi distribution,

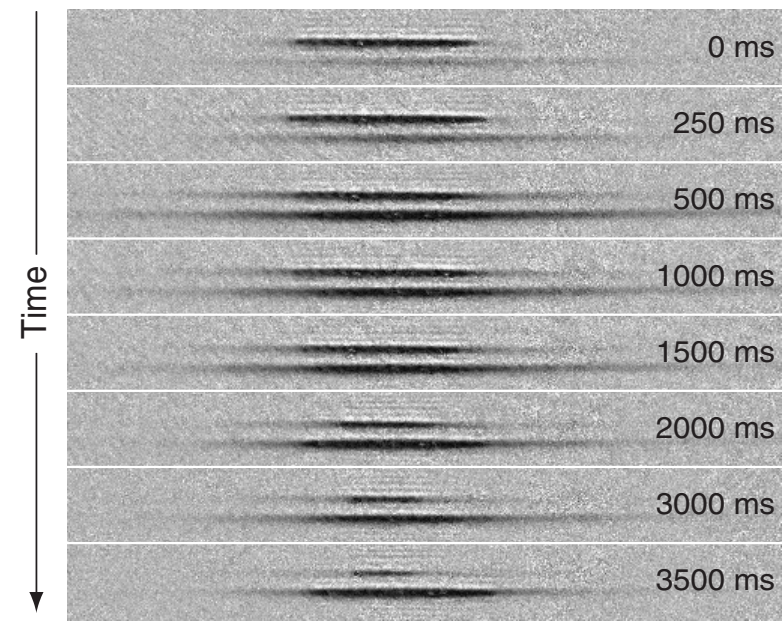

FIG. 2: Time evolution of atom clouds in a double-well potential. The left (right) well appears as the top (bottom) atom cloud in the images. A condensate was distilled from the left to the right well. The absorption images were taken for various hold times after creating the right well. The field of view of each absorption image is $130 \mu \mathrm{m} \times 1160 \mu \mathrm{m}$. The trap depths were $U_{L}=k_{B} \times 2.4 \mu \mathrm{K}$ (left well) and $U_{R}=k_{B} \times 2.9 \mu \mathrm{K}$ (right well) with a potential barrier of $V=k_{B} \times 510 \mathrm{nK}$ between them. During the hold time, the radial separation between the potential wells was $d=15.9 \mu \mathrm{m}$.

and for the thermal clouds, the fits to a Bose-Einstein distribution were restricted only to the wings to avoid the distortions due to the mean field repulsion of the condensate [18]. The temperature turned out to be very sensitive to the value of the chemical potential of the thermal clouds. Assuming local equilibrium, we set the chemical potential of the thermal clouds in each well equal to that of the condensates in the same well. In the absence of a condensate, the chemical potential of the thermal cloud was determined by the fit to a Bose-Einstein distribution

Fig. 3] displays the condensed atom number and temperature for the images of Fig. 2 Condensates started to form in the right well after $(400 \pm 150) \mathrm{ms}$ and saturated within $2 \mathrm{~s}$, resulting in $\sim 50 \%$ of the condensate being transferred. The final temperature in the right well was $T_{f} \sim 350 \mathrm{nK}$, which is $\sim 150 \mathrm{nK}$ higher than the initial temperature $T_{i}$. This increase of temperature reflects the energy gained by the atoms when they "fall" into the right potential well which is deeper by $\Delta U=480 \mathrm{nK}$. After $3.5 \mathrm{~s}$, the total number of atoms of the whole system was $N_{f}=(0.6 \pm 0.1) \times 10^{6}$, which is $15 \%$ less than expected for the measured lifetime of $\tau=12.1 \mathrm{~s}$. Evaporative cooling due to finite trap depth may explain both the atom loss and the fact that the temperature increase was much less than $\Delta U$.

Even after $3.5 \mathrm{~s}$ hold time, full global equilibrium was not reached. This can be seen in both the temperature and the condensed atom numbers. As the chemical po- 

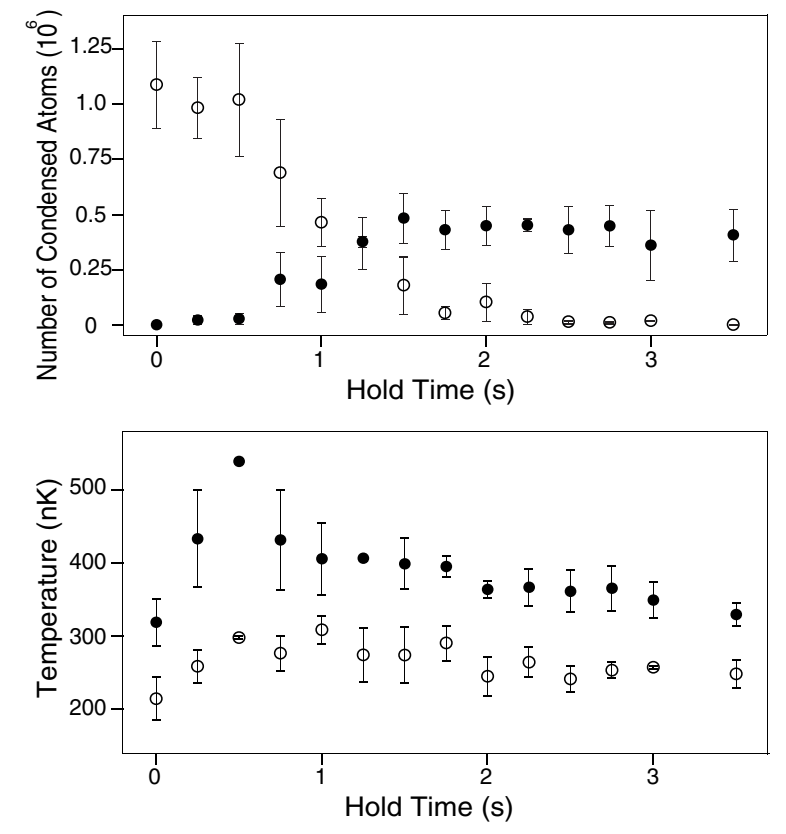

FIG. 3: Approach to thermal equilibrium in a double-well potential. The temperature and the number of condensed atoms in each well are shown as a function of hold time after creating the right well. Open and solid circles represent atoms in the left and the right well, respectively. Every data point is averaged over three measurements, and the error bar shows \pm one standard deviation. The experimental parameters are the same as for the results shown in Fig. 2

tential of condensates in the right well was lower than the trap bottom of the left well, there should not have been any condensate remaining in the left well in global equilibrium. However, Fig. 2] shows a small condensate of $\sim 10^{3}$ atoms in the left well even after 3.5 s holding. Furthermore, the temperature in the left well was measured $\sim 100 \mathrm{nK}$ lower than in the right well.

On first sight, this slow approach towards equilibrium is surprising. In evaporative cooling, one has very fast cooling for a ratio of the height of the potential barrier to the temperature of less than three [19], as was in our experiment. Note, however, that in our trap geometry, the exchange of thermal atoms is geometrically suppressed due to the small "contact area" between the two elongated cigar shaped clouds. Moreover, if the transferred thermal atoms have high angular momentum, they have poor collisional coupling to the cold trapped atoms like the Oort cloud in magnetic traps [20]. Indeed, the density of thermal atoms with higher energy than the potential barrier in the left well after $3.5 \mathrm{~s}$ holding is $\sim 3 \times 10^{11} / \mathrm{cm}^{3}$, and their collision time with the atoms confined in this well is $\left(n \sigma v_{r e l}\right)^{-1} \approx 0.5 \mathrm{~s}$.

Another quantity of interest in the condensate formation process is the onset time of condensation, the hold time until a condensate first appears [4, 5, 21]. To avoid ambiguities in fitting small condensates, we determined

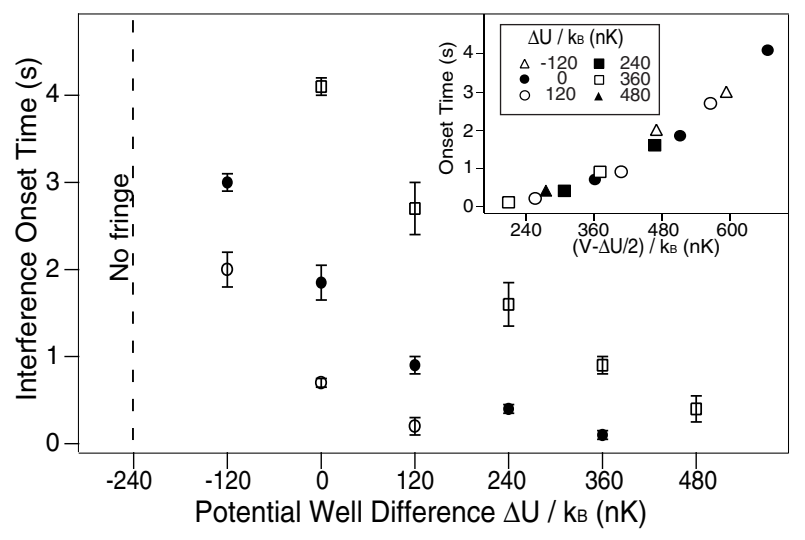

FIG. 4: Onset time of condensation. The onset time in the right well was measured by observing the appearance of a matter wave interference pattern when the condensates were released from the double-well potential. The trap depth difference is defined as $\Delta U=U_{R}-U_{L}$. $U_{L}$ was kept at $k_{B} \times 2.4 \mu \mathrm{K}$ for all experiments. The separations of the two wells, $d$, were $14.3 \mu \mathrm{m}$ (open circle), $15.1 \mu \mathrm{m}$ (solid circle), and $15.9 \mu \mathrm{m}$ (open square). Interference fringes were not observed at $\Delta U=-k_{B} \times 240 \mathrm{nK}$ even after $20 \mathrm{~s}$ hold time. The inset shows the same data plotted vs. $V-\Delta U / 2$ where $V$ is the height of the potential barrier.

the onset time in the right well by observing the appearance of interference fringes when two condensates were released from the double-well potential. For two pure condensates, the visibility of the interference fringes is larger than $55 \%$ as long as the number ratio of the two condensates is larger than $\eta=0.05$. Using the methods described in Ref. [17], we have observed discernible interference fringes down to $\eta=0.08$, corresponding to $\sim 8 \times 10^{4}$ condensed atoms in the right well.

Onset times were measured as a function of $d$ and $\Delta U$ (Fig. 44). The condensate formation is driven by the potential well difference $\Delta U$, whereas the barrier of height $V$ provides the 'resistance' against equilibration, since thermal atoms must have a kinetic energy larger than $V$ to transfer from the left well to the right well. Phenomenologically (see inset of Fig. (4) the condensate onset time depends only on the combination $(V-\Delta U / 2)$ with an almost exponential dependence. $(V-\Delta U / 2)$ can be considered as $\left(V_{\text {eff }}-\Delta U\right)$, where $V_{\text {eff }}=[V+(V+\Delta U)] / 2$ is the average height of the barrier measured from each well.

In two limiting cases, no interference patterns were observed. When the trap depth difference is larger than the peak atomic mean field energy of condensates, i.e. $|\Delta U|>\tilde{\mu}_{0}$, it is energetically favorable for condensates to remain in the lowest well. We observed no interference pattern when $\Delta U=-k_{B} \times 240 \mathrm{nK}$ even after $20 \mathrm{~s}$ hold time. The disappearance of interference fringes was observed when $\Delta U \geq k_{B} \times 360 \mathrm{nK}$ due to complete distillation of the condensates into the right well. In the limit 
where the barrier height is smaller than the peak atomic mean field energy of condensates, i.e. $V<\tilde{\mu}_{0}$, condensate atoms can 'spill' over the potential barrier. Indeed, we observed that condensates appeared in the right well immediately for $V$ less than $\sim k_{B} \times 290 \mathrm{nK}$, consistent with $\tilde{\mu}_{0} \sim k_{B} \times 300 \mathrm{nK}$.

To observe quantum tunneling, the thermal relaxation time $\tau_{t h}\left(\propto \exp \left[V / k_{B} T\right]\right)$ should be longer than the tunneling time $\tau_{t u}\left(\propto \exp \left[\sqrt{V / m \hbar^{2}} w\right]\right)$ where $w$ is the thickness of the barrier. For a thick barrier like ours $(>5 \mu \mathrm{m})$, the tunneling time is extremely long $\left(>10^{5} \mathrm{~s}\right)$ and thermal relaxation is likely to dominate. A high and thin barrier is necessary to observe tunneling and the related Josephson effects.

In conclusion, we have created Bose-Einstein condensates in a metastable state in a double-well potential and studied the dynamical evolution. The observed distillation process is important for equilibration in spinor condensates and for replenishing condensates in continuous atom lasers.

This work was funded by ARO, NSF, ONR, and NASA. We thank $\mathrm{K}$. $\mathrm{Xu}$ for a critical reading of the manuscript. M.S. acknowledges additional support from the Swiss National Science Foundation.

* URL: http://cua.mit.edu/ketterle_group/

[1] P. Nozières, in Bose-Einstein Condensation, edited by A. Griffin, D. W. Snoke, and S. Stringari (Cambrige University Press, Cambride, 1995).

[2] T.-L. Ho, Phys. Rev. Lett. 81, 742 (1998).

[3] T. Ohmi and K. Machida, J. Phys. Soc. Jpn. 67, 1822 (1998).

[4] C. W. Gardiner, M. D. Lee, R. J. Ballagh, M. J. Davis, and P. Zoller, Phys. Rev. Lett. 81, 5266 (1998).

[5] M. Köhl, M. J. Davis, C. W. Gardiner, T. W. Hänsch, and T. Esslinger, Phys. Rev. Lett. 88, 080402 (2002).
[6] H.-J. Miesner, D. M. Stamper-Kurn, J. Stenger, S. Inouye, A. P. Chikkatur, and W. Ketterle, Phys. Rev. Lett. 82, 2228 (1999).

[7] D. M. Stamper-Kurn, H.-J. Miesner, A. P. Chikkatur, S. Inouye, J. Stenger, and W. Ketterle, Phys. Rev. Lett. 83, 661 (1999).

[8] H. Schmaljohann, M. Erhard, J. Kronjäger, M. Kottke, S. van Staa, K. Arlt, J. Bongs, and K. Sengstock, condmat/0308281 (2003).

[9] M.-S. Chang, C. D. Hamley, M. D. Barrett, J. A. Sauer, K. M. Fortier, W. Zhang, L. You, and M. S. Chapman, cond-mat/0309164 (2003).

[10] A. Smerzi, S. Fantoni, S. Giovanazzi, and S. R. Shenoy, Phys. Rev. Lett. 79, 4950 (1997).

[11] S. Giovanazzi, A. Smerzi, and S. Fantoni, Phys. Rev. Lett. 84, 4521 (2000).

[12] L. Pitaevskii and S. Stringari, Phys. Rev. Lett. 87, 180402 (2001).

[13] M. Holland, K. Burnett, C. Gardiner, J. I. Cirac, and P. Zoller, Phys. Rev. A 54, R1757 (1996).

[14] A. P. Chikkatur, Y. Shin, A. E. Leanhardt, D. Kielpinski, E. Tsikata, T. L. Gustavson, D. E. Pritchard, and W. Ketterle, Science 296, 2193 (2002).

[15] F. Dalfovo, S. Giorgini, L. P. Pitaevskii, and S. Stringari, Rev. Mod. Phys. 71, 463 (1999).

[16] T. L. Gustavson, A. P. Chikkatur, A. E. Leanhardt, A. Görlitz, S. Gupta, D. E. Pritchard, and W. Ketterle, Phys. Rev. Lett. 88, 020401 (2002).

[17] Y. Shin, M. Saba, T. A. Pasquini, W. Ketterle, D. E. Pritchard, and A. E. Leanhardt, cond-mat/0306305 (2003).

[18] M. Naraschewski and D. M. Stamper-Kurn, Phys. Rev. A 58, 2423 (1998).

[19] W. Ketterle and N. J. van Druten, Adv. At. Mol. Opt. Phys. 37, 181 (1996).

[20] E. A. Cornell, J. R. Ensher, and C. E. Wieman, in Proceedings of the International School of Physics - Enrico Fermi, edited by M. Inguscio, S. Stringari, and C. E. Wieman (IOS, Amsterdam, 1999).

[21] H.-J. Miesner, D. M. Stamper-Kurn, M. R. Andrews, D. S. Durfee, S. Inouye, and W. Ketterle, Science 279, 1005 (1998). 